The Basic Colour Terms of Ndebele

Author(s): Ian Davies, Christine Davies and Greville Corbett

Source: African Languages and Cultures, Vol. 7, No. 1 (1994), pp. 36-48

Published by: Taylor \& Francis, Ltd.

Stable URL: http://www.jstor.org/stable/1771749

Accessed: 06-02-2017 12:11 UTC

JSTOR is a not-for-profit service that helps scholars, researchers, and students discover, use, and build upon a wide range of content in a trusted digital archive. We use information technology and tools to increase productivity and facilitate new forms of scholarship. For more information about JSTOR, please contact support@jstor.org.

Your use of the JSTOR archive indicates your acceptance of the Terms \& Conditions of Use, available at http://about.jstor.org/terms

Taylor \& Francis, Ltd. is collaborating with JSTOR to digitize, preserve and extend access to African Languages and Cultures 


\title{
THE BASIC COLOUR TERMS OF NDEBELE*
}

\author{
Ian Davies, Christine Davies and Greville Corbett
}

\section{Abstract}

We report a field study of the colour terms of the Bantu language Ndebele. The main purposes of the study were to describe the Ndebele colour term inventory, and to determine which colour terms were basic in order to test Berlin \& Kay's theory of colour universals. A sample of school children and a sample of adults from Matabeleland performed a list task ('tell me as many colour terms as you know') in order to identify Ndebele colour terms and to establish the relative salience of the terms. The adults also took part in a low resolution colour mapping task which provided a preliminary estimate of the range of referents of each term. Our results show that Ndebele has four definite basic terms: terms for white, black, red and green with blue (grue). In addition, the children had a basic term for yellow, and as this term was taught formally in school, it may eventually become basic for the majority of the population. Both sets of basic terms are consistent with Berlin \& Kay's theory.

\section{Introduction}

Colour perception and categorization have been natural testing grounds for theories about the cultural relativity of perception and cognition since at least Rivers (1901). Initially, the variability across languages in the number of colour terms and in what they denoted, was regarded as prima facie evidence for the arbitrariness and relativity of perceptual categories in general (Gleason 1961). However, since Berlin and Kay (1969), the prevailing belief has shifted from such extreme cultural relativism, towards belief in colour 'universals'. They proposed that, rather than varying without constraint colour term inventories were drawn from a severely constrained sub-set represented on the hierarchy shown in Figure 1.

\footnotetext{
* This work was supported by the ESRC grant number R000 23 3978. We gratefully acknowledge this support. We are particularly grateful to Mr Clive Nyoni Nkoomadzinja, the headmaster of Gwaai River School and his staff for their welcome, and for their thoughtful discussions and advice on appropriate translations of Ndebele terms.
} 
FIGURE 1: The Berlin and Kay Hierarchy of basic colour terms

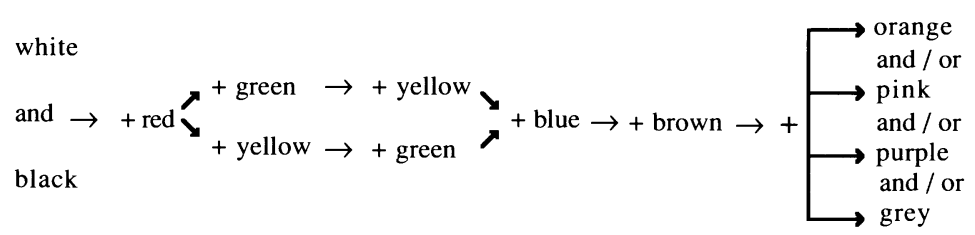

According to the theory, there is an evolutionary progression from a state with just two colour terms (white and black) through to eleven colour terms (all the terms on the hierarchy) by taking the 'permissible routes' described by left to right progression on the hierarchy.

The concept of basic colour term is central to the theory. Basic colour terms are: mono-lexemic, by which Berlin \& Kay mean that their meaning cannot be derived from the meanings of the parts; have unrestricted referents; are not subordinate to another colour term; and are psychologically salient. Basic colour terms must be drawn from the hierarchy, whereas languages may show considerable variation in their non-basic colour terms. Although the theory rests on the idea of basicness, the concept has been criticized as unnecessarily restrictive by Crawford (1982) and Moss (1989).

Kay \& McDaniel (1978) strengthened the theory by linking it to visual perception and physiology. They distinguished between the 'primary' terms: the first six terms on the hierarchy, and the 'derived' terms: the last five terms, by claiming that the primary terms denoted Hering's (1920) six opponent process colours, whereas the derived terms denote 'blends' of pairs of the primary colours. The primary colours are perceptually salient and seem to have unique and independent neural channels, at least in the mid-brain (see, for example, Jameson 1985). Thus, some of the order on the hierarchy was accounted for by saying that colours would be encoded by languages in order of their perceptual salience.

According to Kay \& McDaniel, languages with fewer than six basic terms should have at least one 'composite' category - a combination of two or more primary categories. Thus rather than leaving areas of colour space outside the category system, all of colour space was subsumed under whatever basic terms a language possessed. For instance, the two basic colour terms of the Dani of New Guinea designated light and cool colours on the one hand, and dark and warm colours on the other, (Heider 1971), so that between them the two terms denoted all possible colours. Similarly, many languages, including the languages of southern Africa, have a composite term for the green-blue region of colour space, namely 'green with blue', called 'grue' in the literature (see for example, Davies, MacDermid, Corbett, McGurk, Jerrett, Jerrett \& Sowden 1992, on Setswana). 
Some exceptions to the Berlin and Kay theory have been found, and there are objections to it (see for example Ratner 1989); as a result it has been further developed (see Kay, Berlin and Merrifield 1991), and has proved to be a remarkably robust generalization. However, there have been relatively few studies of the languages of Africa which have included adequate numbers of speakers in their samples. We have reported on Setswana, Xhosa and Chichewa, which are all members of the Bantu group within NigerKordofanian. These three languages all have basic terms for white, black, red and grue; Chichewa has a basic term for yellow, but Setswsana and Xhosa probably do not. There are signs that the composite grue term is splitting into separate terms for blue and green in Chichewa and Xhosa, by means of importing the English term for blue, leaving the grue term to denote green; there was no evidence for such decomposition of the grue term in Setswana.

The present paper reports a preliminary study of Ndebele, the main language of southern Zimbabwe (Matabeleland). It is a Bantu language closely related to Xhosa. The methods we used were not ideal, but were fashioned to fit the circumstances. Nevertheless, in view of the rapidity of change which many southern African languages are exhibiting (see, for instance, Davies, Corbett, McGurk \& Jerrett 1994, on the difference between Setswana spoken by children and adults) and the internal consistency of our results, it is important that we report our findings. Our main method was the list task; this is a simple, but reliable method for eliciting contenders for the basic colour term slots from informants (see Corbett \& Davies 1994, for a discussion of measures of basicness). Basic terms tend to be offered by most informants, whereas secondary terms tend to be offered by relatively few informants. Basic terms also tend to be offered early in informants' lists due to their psychological salience (Berlin \& Kay's fourth criterion). The list task was supplemented by an informal mapping procedure; this provides a low resolution map of colour space, but one which is sufficient to indicate whether the colour terms include any composite terms.

We studied two samples of native Ndebele speakers in Matabeleland; a sample of children aged between 11 and 14 years, and a sample of adults, using the list task for both samples and the mapping task on the adults. In addition we made some informal observations of a younger group of children being taught Ndebele colour terms by their teacher.

\section{Method}

\subsection{Subjects}

There were 34 children in the group that were observed being taught about colours, 19 boys and 15 girls; they were in form one of the school and their ages ranged from six to nine years with a mean of 7.2 years. There were 30 
children who did the list task, 18 boys and 12 girls; they were in form four of the school and their ages ranged from 11 to 14 years with a mean of 12.5 years. Both groups of children attended a rural primary school in central Matabeleland; with one exception all children spoke Ndebele as their first language and Ndebele was the main medium for teaching in the school, although they were also taught English. The child who did not speak Ndebele as her first language spoke Shona, and she performed the list task in Shona; her results have not been included in the data reported here.

There were 39 people in the sample of adults, 27 men and 12 women; their ages ranged from 18 to 57 years with a mean age of 33 years. The sample was drawn approximately equally from two regions of Matabeleland, Gwaai river village in central Matabeleland and Victoria Falls in the north-west of Matabeleland. The first of these regions is dominated by Ndebele speakers, but there is a wider range of languages spoken in Victoria Falls because many people have moved there to work in tourism. All of our sample spoke Ndebele as their first language, but most also spoke English with varying degrees of fluency. The majority of the sample (27) were employed, most commonly as agricultural workers, clerical workers, school teachers and in tourism.

\subsection{Procedure}

\subsubsection{The observation exercise}

Two observers attended a lesson on colour which was given to the first form by the regular form teacher. The teacher showed the children five coloured plastic shapes, one at a time and asked the class, in Ndebele, what colour each object was called. After they had responded, the teacher said the appropriate term herself, and then the children were told to go and point to something in the room of that colour. They then returned to their seats and the next object was presented. The teacher presented the colours in the order: yellow, green, blue, red and orange; the colours were good examples of the English terms: yellow, green, blue, red and orange.

The teacher then stuck the plastic shapes on to the blackboard and asked the children to copy the shapes and to colour the shapes in with the appropriate crayon; in addition to crayons of the five colours given above there were also brown, pink and black crayons available. The teacher wrote the Ndebele and the English name next to each of the colours, except orange, in which case she only wrote the English term (she explained to the observers that there was no term for orange in Ndebele). The children were told to copy the colour terms. 


\subsubsection{The list task: children.}

The teacher told the class in Ndebele to write down as many words for colours as they knew and to number the words in the order they wrote them. The children took from two to ten minutes to complete the task. The teacher then went through the lists with the observers, correcting any spelling mistakes, translating the terms and occasionally asking a child what was meant by a particular term.

\subsubsection{The list task: adults}

Two first-language Ndebele speakers acted as experimenters in collaboration with one of the authors. Respondents were asked in Ndebele to say as many colour words as they knew and these were written down by the Ndebele speaking experimenters. The respondents were encouraged to continue if they stopped and this phase of the investigation finished when the respondents said they were sure they knew no more colour terms. The task took two to three minutes on average.

\subsubsection{Colour mapping}

They were then shown a number of coloured items in order to arrive at an estimate of where the boundaries of the colour terms lay. The colours were good examples of the eleven universal categories, plus a number of intermediate colours: a dark pink verging on red, a dark purple verging on red, a dark blue, a light brown and a yellowy orange. Not all colours were shown to all respondents. The colours selected for particular respondents were chosen partly on the basis of terms 'missing' from their lists; for instance, if a respondent did not offer a brown term they would be shown a good brown and asked what they called it. In most cases two or three focal colours and the 'marginal' colours were shown to each respondent.

\section{Results}

The glosses we give for the colour terms given by our informants are based on several, usually consistent, sources. First, our colleagues in the school spoke fluent English as well as being first language Ndebele speakers. Appropriate translations were discussed with them and the range of colour terms' signification informally explored using available colour samples. In some cases these colleagues asked the children for clarification of some of the less common terms; they also corrected the spelling of the children's lists. We have in general relied upon their expertise, particularly for terms not found in standard dictionaries. Second, our collaborators who collected the adult data 
also spoke English and their translations were generally consistent with those of the school teachers. Third, we have used the data from our various colour term mapping procedures. Fourth, we have used Pelling's (1971) EnglishNdebele dictionary, A Practical Ndebele Dictionary. In general these sources provide consistent glosses, but in a few cases, most significantly for the term kuluhlaza, our other sources were partially at odds with Pelling (1971): Pelling glosses the term as 'green', whereas our other sources suggest strongly that its range includes blue and that therefore 'green with blue' ('grue') is a more appropriate gloss. Although we give the glosses informed by our full set of results in the tables, the justification for some of them may not be apparent until the end of the results section.

\subsection{The observation exercise}

(1) Yellow: about half of the children shouted kulithanga 'yellow' in response to the yellow object; most of the class then pointed at yellow things such as pictures of bananas, but some pointed at orange things such as pictures of oranges.

(2) Green: about three-quarters of the class shouted kuluhlaza 'green with blue' ('grue') to the green object and most of them pointed at green objects and some at blue objects.

(3) Blue: less than a quarter responded to the blue object, and it was not possible to judge what the responses were. The teacher told them that the name was isibhakabhaka 'blue' (literally as blue as the horizon, or sky). The colours of the objects they pointed to were very varied; these included greens, a few blues some light browns (chickens eggs), and it was not possible to establish the modal response.

(4) Red: virtually all the children responded kubomvu 'red', very loudly, to the red object and pointed mostly at red objects, but some pointed at either pink or orange objects.

(5) Orange: there was little response to the orange tile; a few said kubomvu 'red' and a few said pinki 'pink'. They pointed at oranges and yellows about equally, but a wide range of other colours were also pointed at.

In copying the shapes and colouring them in, the children mostly chose the right crayons for the red, green and blue shapes; but orange and yellow crayons were used about equally for the yellow and orange objects.

In summary, across the three kinds of colour task: colour naming, colour comprehension and colour copying, the relative order with which the children could cope with the colours was: red, green, yellow, blue and orange. 


\subsection{List task: children}

On average the children offered 8.3 terms, and boys and girls offered about the same number of terms: mean for the boys $=8.4$; mean for the girls $=8.1$. There were 27 different terms offered in total, and these are shown in table 1 together with their English gloss; in some cases the colour-meaning of a term derives from the colour of an object denoted by the term, in which cases we give both the colour gloss and the name of the source object. Table 1 also shows the number of children who offered each term (the frequency) and the mean list position for each across those children that offered that term; the terms are ordered by frequency with the most common terms given first. The root form of the terms can take a number of prefixes such as ku-, and oku- . We have given the most frequent form that each term was offered in. There were five terms that were each offered by a clear majority of the sample (at least 90\%): kumhlophe 'white', kumnyama 'black', kubomvu 'red', kuluhlaza 'grue' and kulithanga 'yellow'. The first four of these terms also had the four highest list positions and the fifth, kulithanga 'yellow', shared the seventh highest position. The next most frequent term, khaki 'khaki', was offered by less than half of the sample.

1 One informant said that the prefix ku-with a root colour term such as mhlophe 'white' could be translated as 'that which is white' or 'whiteness'; and the prefix oku-meant 'like the colour of'. 
TABLE 1: Lists - Children

Terms offered by the children, their frequency and mean position

$\begin{array}{llcc}\text { Term } & \text { Gloss } & \text { Frequency } & \text { Position } \\ \text { kubomvu } & \text { red } & 29 & 2.4 \\ \text { kumhlophe } & \text { white } & 28 & 4.1 \\ \text { kumnyama } & \text { black } & 28 & 3.1 \\ \text { kuluhlaza } & \text { grue } & 26 & 3.0 \\ \text { kulithanga } & \text { yellow } & 26 & 5.3 \\ \text { khaki } & \text { khaki } & 17 & 6.4 \\ \text { bulu } & \text { blue } & 15 & 6.5 \\ \text { ilubende } & \text { brown (cooked blood) } & 13 & 6.6 \\ \text { kyelo } & \text { yellow } & 11 & 5.3 \\ \text { igilini } & \text { green } & 8 & 6.9 \\ \text { imeroni } & \text { maroon } & 8 & 7.0 \\ \text { igrey } & \text { grey } & 7 & 7.6 \\ \text { okuyisibhakabhaka } & \text { blue (sky) } & 7 & 5.0 \\ \text { browni } & \text { brown } & 6 & 7.2 \\ \text { kulidube } & \text { striped (zebra) } & 6 & 8.7 \\ \text { luthuli } & \text { grey (dust) } & 5 & 7.0 \\ \text { Okulithendele } & \text { spotted (guinea fowl) } & 4 & 8.3 \\ \text { pinki } & \text { pink } & 4 & 9.3 \\ \text { lithubi } & \text { brown (egg-yolk) } & 3 & 7.7 \\ \text { okuyimpunga } & \text { grey (hair) } & 3 & 5.7 \\ \text { umdaka } & \text { brown (mud) } & 2 & 7.5 \\ \text { okungumherwazi } & \text { black and white } & 1 & 5.0 \\ \text { okuintwala } & \text { grey } & 1 & 11.0\end{array}$

Most of the 12 next most frequent terms (frequencies ranging from 4 up to 15) were 'borrowed' English terms such as bulu 'blue', kyelo 'yellow' and igilini 'green'. Terms which are at least partially equivalent to most of these English imports do exist, and were offered by some of the children. But in general, the English imports were used as additional terms, rather than alternative terms: 10 out of the 11 children who offered iyelo 'yellow', also offered kulithanga 'yellow', and 7 out of 8 offered both igilini 'green' and kuluhlaza 'grue'. This pattern of offering both terms was less strong for the two pairs: browni 'brown' and ilubende 'brown' and bulu 'blue' and okuyisibhakabhaka 'blue'; both terms were offered with about half their possible maximum conjoint frequency. One borrowed term, igrey 'grey', did have a relationship of mutual exclusivity with its traditional partner luthuli 'grey', in that they were never offered by the same person. There were also two borrowed terms that were offered a few times, imeroni 'maroon' and pinki 'pink', which have no real traditional equivalent. 
There were three terms offered all of which we have glossed as 'brown'; these were: ilubende (cooked blood), lithubi (egg-yolk), and umdaka (mud). Pelling (1971) glosses lithubi as 'yellow', but the only children to offer lithubi also offered kulithanga 'yellow', whereas the three 'brown' terms were offered more or less mutually exclusively — only one child offered more than one of them - suggesting that they might be alternatives for brown.

There were three terms offered which denoted colour combinations or perhaps surface texture rather than uniform colour: okulithendele 'speckled' (literally 'like a guinea fowl'), kulidube 'black and white' (like a zebra) and okungumherwazi 'black and white'. The frequency of these mixed terms was low.

\subsection{List task: adults}

There were 20 different terms offered by the adults and the mean number of terms offered was 5.1. Men and women offered about the same number of terms (means: men $=5.1$, women $=5.0$ ), but the adults offered fewer terms than the children ( mean $=8.3$ ).

The terms offered by the adults are shown in table 2 together with their English gloss, their frequency and mean list position; they are given in order of their frequency. The five most frequent terms are the same as the five most frequent terms given by the children: kumhlophe 'white', kumnyama 'black', kubomvu 'red', kuluhlaza 'grue' and kulithanga 'yellow'. But, while these five terms were offered by about $90 \%$ of the children, they were offered by between about $50 \%$ and $85 \%$ of the adults. The largest difference was for the term kulithanga 'yellow' which was offered by about $90 \%$ of the children but by only about $50 \%$ of the adults. The adults, unlike the children, did not in general offer borrowed terms; the single exception was bulu 'blue'. 
TABLE 2: Lists - Adults

Terms offered by the adults, their frequency and mean position

$\begin{array}{llcl}\text { Term } & \text { Gloss } & \text { Frequency } & \text { Position } \\ \text { kubomvu } & \text { red } & 33 & 2.2 \\ \text { kuluhlaza } & \text { grue } & 32 & 2.5 \\ \text { kumhlophe } & \text { white } & 31 & 2.5 \\ \text { kumnyama } & \text { black } & 25 & 2.9 \\ \text { kulithanga } & \text { yellow } & 19 & 3.2 \\ \text { ilubende } & \text { brown (cooked blood) } & 8 & 4.8 \\ \text { luthuli } & \text { grey (dust) } & 8 & 4.5 \\ \text { okuintwala } & \text { grey } & 6 & 4.2 \\ \text { lithubi } & \text { brown (egg-yolk) } & 4 & 4.8 \\ \text { okulithendele } & \text { spotted (guinea fowl) } & 5 & 3.6 \\ \text { okuyimpunga } & \text { grey (hair) } & 4 & 5.5 \\ \text { okuyisibhakabhaka } & \text { blue (sky) } & 3 & 4.7 \\ \text { bulu } & \text { blue } & 3 & 5.7 \\ \text { umdaka } & \text { brown (mud) } & 3 & 5.7 \\ \text { okungumherwazi } & \text { black and white } & 3 & 4.0 \\ \text { okugamane } & \text { yellow (butterfly) } & 2 & 2.5 \\ \text { okulizulu } & \text { blue (sky) } & 2 & 5.5 \\ \text { okungumiwo } & \text { brown } & 1 & 3.0 \\ \text { okuvuithiwego } & \text { grey } & 1 & 2.0 \\ \text { okuliganu } & \text { yellow (fruit) } & 1 & 4.0\end{array}$

There were three terms offered which we have glossed as 'grey': luthuli, okuintwala, and okuyimpunga. They were used almost mutually exclusively; just one person offered more than one of them. There were also three brown terms offered - ilubende, lithubi, and umdaka - which were offered mutually exclusively, which supports the suggestion in the children's lists section that they might be alternatives for brown.

The last five terms in table 2, terms with frequencies of either 1 or 2 , were terms not offered by the children. They are all the colours of objects as indicated by the prefix oku-.

\subsection{Colour Mapping}

All informants named focal white by the term kumhlophe 'white', focal black by kumnyama 'black', focal red by kubomvu 'red', and focal green by kuluhlaza 'grue'. There was less clear agreement for focal blue: 17 people said it was kuluhlaza 'grue', seven said it was either okuyisibhakabhaka 'sky' or okulizulu 'sky', five said it was bulu 'blue' and the rest said they 
did not know. Focal yellow was called kulithanga 'yellow' by 20 people, okuugamaneevane 'yellow butterfly' by one person and the rest did not name it. Focal brown was called by the various brown terms: ilubende by ten people, umdaka by two people and lithubi by two people. Focal pink, orange and purple were in general not named with a traditional Ndebele word, but a few informants offered the appropriate English terms; when directly asked whether they were included in kubomvu 'red', kulithanga 'yellow', or kuluhlaza 'grue', virtually all informants said they were not. However, dark pink and reddish purple were included in kubomvu 'red' by most people; dark blue and dark brown were included in kumnyama 'black' by the majority of informants, and ten people included bluish purple in kuluhlaza_'grue'. Pale colours such as, light grey and yellow, were not included in 'white' by anyone. Medium grey was called luthuli 'grey' by five people and okuyimpunga 'grey' by two people.

\section{Discussion}

Four terms - kumhlophe 'white', kumnyama 'black', kubomvu 'red' and kuluhlaza 'grue' - show the high level of salience shared by the majority of respondents which is characteristic of basic terms. These terms were offered by the great majority of the sample and they tended to be amongst the first terms offered. Further, the observation of the youngest children, the informal mapping procedure and the translations offered by our Ndebele speaking colleagues, all converge to indicate that these terms correspond to the universal terms white, black, red and grue. Their foci are at least close to the universal foci, but their range is somewhat wider than indicated by the English glosses. This extended range can be seen most clearly for kuluhlaza 'grue', which has the main characteristics of a composite term: most particularly its range includes the range of the two primary terms green and blue. Even so, kuluhlaza 'grue' is more strongly focused in the green region than the blue region. This was suggested by the mapping procedure, the translations of our colleagues, and perhaps most significantly, by the way colour terms were being taught in school. There, the children were being taught separate terms for green and blue: kuluhlaza was taught as green and isibhakabhaka as blue. Thus as with Chichewa (Davies, Corbett \& Mtenje forthcoming) and Xhosa (Davies \& Corbett forthcoming) there is evidence that the grue term is decomposing into two primary categories. In the latter cases, this seems to be happening by leaving the grue term to denote green and using the borrowed term bulu, or blue, or blau to denote blue. The process in Ndebele seems to be similar except that it may be the traditional term okuyisibhakabhaka (sky) that becomes the basic term for blue. Our uncertainty here is because, although the younger children were taught okuyisibhakabhaka as blue, many of the older children used the borrowed term bulu. 
There was some suggestion that other terms had broader ranges than their glosses might imply: 'black' included dark greys and blues; kubomvu 'red' included reddish purples and dark pinks; and kulithanga 'yellow' (even though its status as a basic term is not as clear as the four terms we have so far discussed) includes some orange colours. On the other hand, these terms did not appear to have the complete characteristics of composite terms; rather than sharing out all of colour space between them, they seemed to include the nearest regions of their 'missing' neighbours, but their domain did not extend far enough to include the missing foci. For instance, kubomvu 'red' did not include focal pink. Our conclusions here must be tentative, because of the informal nature of the mapping task, and the very coarse sampling of colour space used, but we found a similar pattern in Setswana, Davies et al. (1992) and Xhosa, Davies \& Corbett (forthcoming); in both cases there were large areas of colour space around the missing foci that our informants did not name. Our data on Chichewa, (Davies et al. forthcoming), showed more evidence of composite categories as envisaged by Kay \& McDaniel (1978), but even in Chichewa, our informants distinguished between focal examples of the composite categories and more distant examples by using an attenuated form of the colour term for the more distant colour. For instance, good reds were called chobiriwira 'red', whilst pinks, reddish purples and reddish oranges were called chobiriwirira 'reddish'.

In addition to the four terms we have discussed so far, there is a further term, kulithanga 'yellow', which may also be basic. It showed the same pattern of 'frequent salience' as the four terms already discussed, in the data from the children, although for the adults it was a less prevalent term than the other four. The greater incidence of the term amongst the children may be due to how they are taught in school, or it might be because the children wrote their responses whereas the adults spoke theirs. The children thus had more chance to deliberate, and this is probably why they produced more terms on average than the adults. Whatever the reason for the greater prevalence of kulithanga 'yellow' amongst the children, if they are a typical group of children, then it is likely that kulithanga 'yellow' will eventually become basic for the whole population.

\section{Conclusion}

Ndebele probably has four basic colour terms: kumhlophe 'white', kumnyama 'black', kubomvu 'red' and kuluhlaza 'grue'. Kuluhlaza 'grue' has the characteristics of a composite category, but we may be observing its decomposition into separate green and blue terms. To a lesser extent, kumnyama 'black' and kubomvu 'red' also have the characteristics of composite categories but their range does not seem to extend as far as the foci of their 'missing' neighbouring categories. Kulithanga 'yellow' may be basic for 
children, and may become basic for the whole population. These conclusions need checking with a more detailed mapping procedure to establish the range and focus of each term.

\section{REFERENCES}

Berlin, B. \& P. Kay. 1969. Basic Color Terms: their Universality and Evolution. Berkeley \& Los Angeles: University of California Press.

Corbett, G.G. \& I.R.L. Davies. 1994. Linguistic and behavioural measures for ranking basic colour terms. Studies in Language in press.

Crawford, T.D. 1982. Defining 'basic color term'. Anthropological Linguistics 24: 338-343.

Davies, I.R.L. \& G.G. Corbett. Forthcoming. The basic colour terms of Xhosa.

Davies, I.R.L.,G.G.Corbett, H. McGurk \& D.J. Jerrett. 1994. A developmental study of colour terms in Setswana. Journal of Child Language in press.

Davies, I.R.L., G.G. Corbett \& A. Mtenje. Forthcoming. The basic colour terms of Chichewa. Lingua in press.

Davies, I.R.L., C. Macdermid, G.G. Corbett, H. McGurk, D. Jerrett, T. Jerrett, \& P. Sowden. 1992. Color terms in Setswana: a linguistic and perceptual approach. Linguistics 30: 1065-1103.

Gleason, H.A. 1961. An Introduction to Descriptive Linguistics. New York: Holt, Rinehart and Winston.

Jameson, D. 1985. Opponent-colours theory in the light of physiological findings. In D. Otteson \& S. Zeki (eds.) Central and Peripheral Mechanisms of Colour Vision. London: Macmillan.

Hering, E. 1920. Outlines of a Theory of the Light Sense. Translated from German by L.M. Hurvich \& D. Jameson 1964. Cambridge: Harvard University Press.

Heider, E.R. 1971. 'Focal' color areas and the development of color names. Developmental Psychology 4: 447-455.

Kay, P., B. Berlin \& W. Merrifield. 1991. Biocultural implications of systems of color naming. Journal of Linguistic Anthropology 1(1): 12-25.

Kay. P. \& C.K. McDaniel. 1978. The linguistic significance of the meanings of basic color terms. Language 54: 610-646.

Moss, A.E. 1989. Basic colour terms: problems and hypotheses. Lingua 78: 313320.

Pelling, J.N. 1971. A Practical Ndebele Dictionary. Revised edition. Harare: Longman Zimbabwe.

Ratner, C. 1989. A sociohistorical critique of naturalistic theories of color perception. The Journal of Mind and Behaviour 10: 361-372.

Rivers, W.H.R. 1901. Introduction and vision. In A.C. Haddon (ed.) Reports on the Cambridge Anthropological Expedition to the Torres Straits, vol. 2. Physiology and Psychology. Cambridge: Cambridge University Press. 